\title{
Detecting photons with orbital angular momentum in extended astronomical objects: application to solar observations (Research Note)
}

\author{
N. Uribe-Patarroyo ${ }^{1}$, A. Alvarez-Herrero ${ }^{1}$, A. López Ariste ${ }^{2}$, A. Asensio Ramos ${ }^{3,4}$, T. Belenguer ${ }^{1}$, R. Manso Sainz ${ }^{3}$, \\ C. LeMen ${ }^{2}$, and B. Gelly ${ }^{2}$
}

${ }^{1}$ Laboratorio de Instrumentación Espacial (LINES), Instituto Nacional de Técnica Aeroespacial (INTA), Ctra. Ajalvir km 4 , Torrejón de Ardoz, 28850 Madrid, Spain e-mail: uribepnr@gmail.com; alvareza@inta.es

2 THEMIS, CNRS UPS 853, C/ vía Láctea $\backslash$ \, La Laguna, 38205 Tenerife, Spain e-mail: [arturo;bgelly]@themis.iac.es;claude.lemen@gmail.com

3 Instituto de Astrofisica de Canarias, C/ vía Láctea $\backslash \backslash \mathrm{n}$, La Laguna, 38205 Tenerife, Spain e-mail: aasensio@iac.es;rms.iac@gmail.com

4 Departamento de Astrofisica, Univ. de La Laguna, La Laguna, 38205 Tenerife, Spain

Received 22 April 2010 / Accepted 17 October 2010

\section{ABSTRACT}

\begin{abstract}
Context. The orbital angular momentum (OAM) of the photon is a property of light from astronomical objects that has not yet been measured. We propose a method of measuring the OAM spectrum of light from an extended natural source, the Sun. Relating the OAM spectrum of different solar areas to its structures could lead to a novel remote sensing technique.

Aims. We present a method for measuring the OAM spectrum of solar photons.

Methods. The THEMIS ${ }^{\star}$ telescope is being used with a novel phase-diversity technique. A spatial light modulator is placed on one pupil image, and an ad-hoc optical setup allows the measurement of two simultaneous phase-diverse images in the same CCD, with equal optical paths.

Results. Preliminary results show that very good seeing is mandatory for this kind of observation. The method works in the laboratory, and good seeing conditions in the 2010 campaign are being awaited.
\end{abstract}

Key words. instrumentation: miscellaneous - techniques: miscellaneous

\section{Introduction}

It is a known fact that photons carry angular momentum, where the part associated with their spin degree of freedom is classically manifested as the polarization of light, and the orbital part associated with the phase distribution of their wavefronts is manifested as vortices and referred to as the photon's orbital angular momentum (OAM) (For a theoretical account, see Jackson 1962.) However, light with non-zero OAM has only recently been realized experimentally by producing light whose wavefront has an azimuthal phase dependence proportional to $\exp (i l \phi)$ (Allen et al. 1992; Franke-Arnold et al. 2008). This orbital angular momentum is considered to be quasi-intrinsic (see Zambrini \& Barnett 2006), and it has never been measured in a natural source such as an astronomical object, nor has any concrete candidate been proposed, apart from some abstract ideas (Harwit 2003).

The most comprehensive work concerning OAM in astronomy is the extensive paper by Elias (2008), where the central idea is to show how OAM observables are modified by propagation through a generic, aberrated optical system, also discussing how different instrumental strategies affect OAM in a

* THEMIS (Télescope Héliographique pour l'Étude du Magnetisme et les Instabilités Solaires) is a $0.9 \mathrm{~m}$ solar telescope property of the French CNRS-INSU at the Spanish Observatorio del Teide. hypothetical observation. However, the topic of how OAM is generated by astronomical sources is avoided. Although it is not yet clear that natural objects can produce photons with non-zero OAM, an interesting proposal to measure the OAM of point-like astronomical objects has been proposed recently (Berkhout \& Beijersbergen 2009).

Another interesting link between OAM and astronomy that has seen much growth recently, is the use of vortex coronagraphs (Swartzlander 2001; Jenkins 2008; Danchi et al. 2004; Serabyn et al. 2010; Elias 2008), in which the properties of optical vortices are used to design novel types of coronagraphs. However, this employs the OAM of the photon as an observational tool, but not as the quantity intended to be measured.

In this note, we present the preliminary results of the first version of an instrument designed to measure the spectrum of OAM of photons coming from the solar surface. This is a reasonable target, as we will have a large amount of photons at our disposal. However, measuring the OAM of photons coming from the solar surface is conceptually different from measuring the photon OAM spectrum of a point-like astronomical object, because we have to deal with innumerable point-like sources on the surface of the Sun, which inhibits the use of common measurement techniques (Leach et al. 2002; more on this topic in Sect. 3). Therefore, we propose using a novel phasediversity technique (Uribe-Patarroyo et al. 2010b) that allows 
measurement of the OAM superposition state of the photons emitted by an extended object. This technique discerns the OAM content of the photons independently of the spatial structure of the beam.

Since the turbulent atmosphere and the observing instrument modify the spectrum of OAM (see Uribe-Patarroyo et al. 2010a; Tyler \& Boyd 2009; Yi-Xin \& Ji 2009; Gbur \& Tyson 2008; Paterson 2005), we face the challenge, as with any other known technique, of isolating the contribution of the actual measured object to the measured OAM spectrum. It could be the first time in which photons with non-zero OAM are detected in a natural astronomical object, and this could lead to a new kind of remote sensing in astronomy using OAM measurements.

\section{OAM of solar photons}

The measurement of the angular momentum content of light (either coming from extended or point sources) is a powerful tool long used in the case of the spin angular momentum of the photon: polarization. In the case of the Sun, it is known that polarization of light provides invaluable information about its structure, thanks to the relation between the Sun's magnetic fields and the polarization absorption characteristics of the medium (see, e.g., Solanki 1993; Stenflo 1994; Solanki 2003). For this reason, the spin angular momentum of the photon is measured extensively in the field of spectropolarimetry (e.g., del Toro Iniesta 2003) to study magnetic and thermodynamical properties of the solar surface. The following question arises naturally: is there any mechanism by which photons with non-zero orbital angular momentum are produced in the Sun? Could the OAM spectrum be used in a similar fashion to the spin of the photon for remote sensing?

Only recently has there been some work showing how a magnetic field interacts with an atomic system leading to the emission of photons with non-zero OAM. The final result depends on the nature of the magnetic field and the specific atomic spin levels of the system (Akamatsu \& Kozuma 2003). It could be possible that some of those conditions are fulfilled in the Sun, even if for short periods of time and in regions where the spectral lines are out of local thermodynamical equilibrium to avoid collisional thermalization. Another possible source of photons with non-zero OAM are electric quadrupole transitions. In those transitions, the change of angular momentum is one unit more than what can be carried by the polarization of the photon. It could be possible then that the remaining angular momentum is transferred to photon OAM. As a consequence, the measurement of the OAM spectrum of quadrupole spectral lines is a possible target.

If we want to test the hypotheses above, we need to be able to measure the OAM spectrum of solar photons coming from a defined region of the Sun, hoping to fulfill the magnetic field form condition for the first hypothesis and/or observe certain spectral lines whose transition probability is dominated by the quadrupole component. Therefore, it is crucial to consider a technique that allows us to measure the OAM spectrum in an extended object. A novel technique has been recently developed by Uribe-Patarroyo et al. (2010b) for this purpose, and it is explained briefly in Sect. 4.

One characteristic of this method is that it measures the OAM spectrum of a zone of the observed field, but not point-bypoint. The reason is that the phase-diversity algorithm needs a certain number of pixels to correctly estimate the OAM spectrum expansion. It is also expected because the OAM space has infinite dimensions, and even if we limit ourselves to a certain subspace, the amount of experimental points should be greater than the dimension of the OAM subspace being sampled. However, it is possible to subdivide the observed field into small patches inside which the OAM spectrum is measured. Once the OAM spectrum is available for a certain patch, it could be possible to compare it with patches having different magnetic structures, or with the OAM content at different wavelengths. The results of this could enable further studies in the topic to explain the observations, and may even produce a new way of remote sensing.

\section{Challenges of measuring astronomical OAM}

Some difficulties may arise when observing OAMs of astronomical objects. Some of them have already been pointed out in the literature. We summarize them in the following paragraphs.

In Berkhout \& Beijersbergen (2009), the authors propose a method for avoiding the problem of the (possible) extremely low intensity of the vortex beam when it reaches the telescope. This is still an open question, as it is not clear how the multipoint interferometer they propose will improve the situation when dealing with astronomical sources. The distance traveled by the vortex surpasses, by orders of magnitude, any improvement that could come from a realizable array baseline.

Many advances have been made in the field of partially coherent vortex beams (Agarwal 1999; Bogatyryova et al. 2003; Ponomarenko 2001; Palacios et al. 2004; Elias 2008). One of its main consequences for natural sources with OAM is the possibility of having photons with non-zero OAM inside a beam with zero total OAM. As we see in Palacios et al. (2004), it is possible to have low-coherence vortex beams in which the dark core of the vortex is lost in the far field, although the vortex properties are still seen in the cross-correlation function in a plane perpendicular to the propagation direction. This could be the answer to the previous concern: if the OAM state coming from the Sun is a partially coherent superposition, the dark core will no longer exhibit such a low intensity.

Another concern is the alignment of the reference axis of an incoming vortex beam. If a photon with non-zero OAM is actually produced in the region of the Sun we are observing, the axis with respect to which it has a defined OAM is not necessarily the line of sight. It is known that misalignment produces an alteration of the OAM spectrum (Vasnetsov et al. 2005; Elias 2008), although it is clear that the spectrum of an originally non-zero OAM will never be transformed into a symmetric beam carrying zero OAM. However, Liu et al. (2008) have demonstrated that, using the transformation properties of OAM spectrum under misalignment makes it possible to retrieve information about the original spectrum.

\section{Measuring method for extended sources}

The usual techniques for measuring the OAM content of light are not suitable for astronomy (Zambrini \& Barnett 2006), as they cannot be used to carry out measurements faster than the typical turbulent timescale of the atmosphere on the order of $10 \mathrm{~ms}$. Other techniques are only able to measure point-like sources (Leach et al. 2002; Mair et al. 2001). In our case, pointlike measurements cannot be reproduced when remote-sensing an extended object. If one observes with a telescope and places a pinhole in an intermediate focal plane, all the wavefront information associated with that point is lost. The reason is that it acts as a delta function filter in real space, and this translates into a low-pass filter in the Fourier domain, which is precisely the pupil of the system. However, this also gives a hint of how to design 


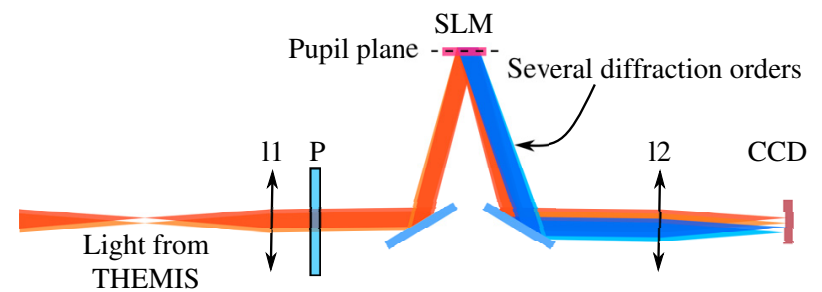

Fig. 1. (Color online) Schematic representation of the THEMIS postfocal instrument. The converging light coming from THEMIS is collimated by lens 11, which also produces an image of the telescope pupil in the SLM aperture. The linear polarizer's P azimuth is aligned with the SLM azimuth. There, a diffraction grating produces three diffraction orders with different vortex phases $(-1,0,+1)$, and those are re-imaged by 12 onto the CCD. Therefore, all the diffraction orders share the same optical path, and the phase diversity algorithm is applied to images taken in different parts of the same CCD.

appropriate techniques. In essence, one has to measure the full wavefront of incoming light to determine whether it is vortexlike. Also, the technique should allow subdivision of the field. Therefore, one of the most straightforward techniques that allow us to estimate the OAM superposition state of the incoming light (Uribe-Patarroyo et al. 2010b) from intensity measurements is phase-diversity; for traditional phase-diversity, see Gonsalves \& Chidlaw (1979) and Paxman et al. (1992).

Schematically, the technique consists of a traditional phasediversity procedure in which the diverse phase consists of the phase pattern of a known vortex beam (instead of the standard defocus). Two (or more) images are taken for different vortex phases and they are treated under a typical phase diversity process. The generalized pupil GP of the observing optical system upon which the error metric depends is conveniently modified as

$G P(\rho, \phi)=W(\rho, \phi)\left(\mathrm{e}^{\mathrm{i} \sum_{n} b_{n} R_{n}(\rho)} \sum_{l} d_{l} \mathrm{e}^{\mathrm{i} l \phi+\mathrm{i} \phi_{l}}\right)$,

in an analogous manner to the treatment of aberrations with Zernike polynomials (Löfdahl \& Scharmer 1994; Bonet 1999). Here, $W(\rho, \phi)$ is the pupil transmission function, a real function describing the apodization nature of the pupil. The factors inside the parenthesis describe the deviation of the wavefront from a spherical wavefront in terms of OAM-Zernike states (see UribePatarroyo et al. 2010a) with real constants $d_{l}$ and $\phi_{l}$. The weight of each OAM mode in the superposition is given by $d_{l}$, and $\phi_{l}$ takes the relative phases between different OAM states into account.

In this case, however, the objective is not that of object restoration to compensate for atmospheric disturbances, but rather the estimation of the optical transfer functions (OTFs) of the system. As in the standard phase-diversity algorithm, the error metric to be optimized depends on the Fourier transform of the images and on the OTF of the system. When the minimum of the error metric is found, the coefficients of the OTF decomposition on the OAM-Zernike basis correspond to the weights for the different OAM modes present in the incoming beam. We want to stress that the determination of the OAM modes comes only from the OTF of the system, as it is manifested in the pointspread function (PSF). Therefore, the spatial structure of the object itself does not affect the measured OAM spectrum. Also, in low numerical aperture (NA) systems like telescopes, the PSF is independent of the polarization of light. Therefore, our determination of the OAM spectrum is independent of the spin content of the photons. This is clearly not true for high NA systems.
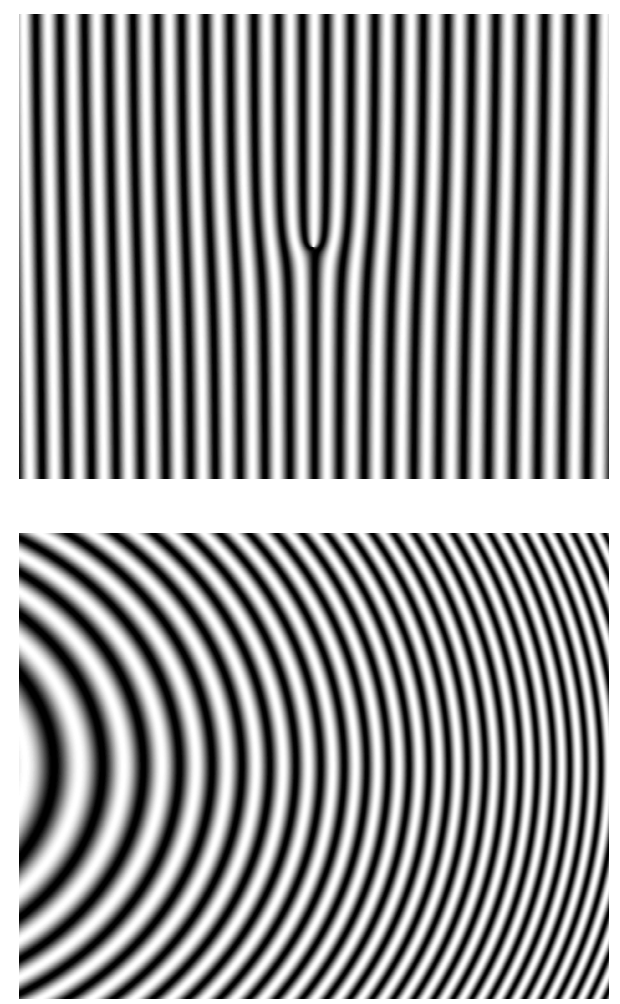

Fig. 2. Diffraction gratings used for phase diversity. Top, vortex diffraction grating produces three main diffraction orders with $(-1,0,+1)$ OAM. Bottom, defocus diffraction grating producing (negatively defocused, focused, positively defocused) diffraction orders, which can be used for traditional phase-diversity.

We modified the TUNIS instrument of THEMIS (Rayrole \& Mein 1993) to accommodate a post-focal instrument for performing this kind of measurements. A schematic design is shown in Fig. 1. We re-imaged the pupil of THEMIS onto the active zone of a liquid crystal (LC) based spatial light modulator (SLM). The LC-based SLM can only introduce an arbitrary phase for one linear polarization, therefore the light entering the SLM must be first polarized with a linear polarizer with the appropriate orientation. This, of course, implies the loss of roughly half the light entering the system. In a more refined version of the instrument, this can be overcome by using the SLM twice for each polarization or by using two SLMs (Vicari 2003, p. 131). The SLM permits the creation of computer-controlled diffraction gratings. We used a common sine diffraction grating (see Fig. 2) for a vortex beam (see Gibson et al. 2004) in which we had three diffraction orders, \pm 1 and 0 OAM. With additional optics, we focused the light onto an iXon ${ }^{\mathrm{EM}}+897 \mathrm{CCD}$, with $16 \mu \mathrm{m}$ pixel size, with $f / 44.8$ (35.5 $\mu \mathrm{m}$ per Airy disc) and $40300 \mathrm{~mm}$ of focal length. That means $2.22 \mathrm{px} /$ Airy, and $0.067 \mathrm{arcsec} / \mathrm{px}$. We want to stress that this technique allows us to measure the OAM spectrum with very short exposure times, provided the images have enough signal-to-noise. Another important property is that the OAM spectrum can be obtained from only a single CCD image. Consequently, we guarantee that the state of the atmosphere in the whole measurement is the same.

\section{Preliminary results}

The 2D spatial images shown in Fig. 3 have, in the $x$ coordinate, an identical wavelength dispersion of about $2.87 \mathrm{~nm}$ for each strip, and the width of each strip corresponds to 10.5 arcsec. 


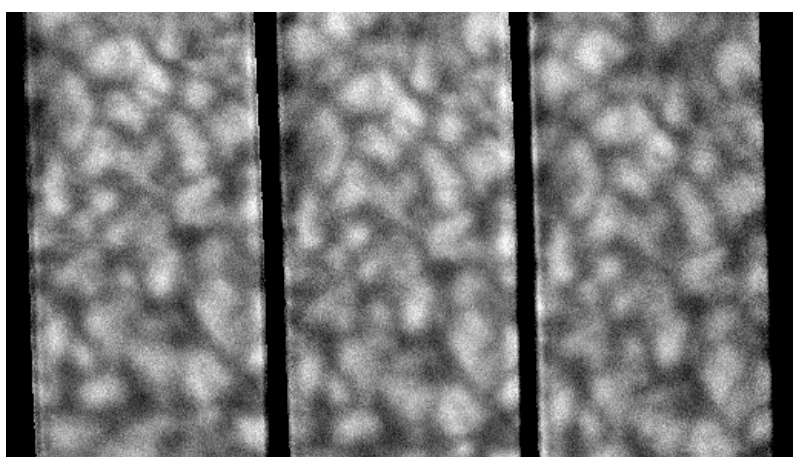

Fig. 3. Unfiltered full CCD images of vortex phase diversity. The central strip is the nominal image, and the left (right) strips the $-1(+1)$ vortex phase-diverse images.

The images have been corrected for dark current and flat field but not filtered by any means. We show the three diffraction orders of the SLM with $(-1,0,+1)$ OAM. For comparison, Fig. 4 shows an example of the images used with traditional phase diversity, showing negatively defocused, focused, and positively defocused diffraction orders from left to right. The amount of defocus was set to about $0.5 \lambda$ at the edge of the pupil. As can be seen, since the seeing was very poor, the nominal images are already too aberrated, and there is not too much difference between them. It is widely known that the phase-diversity technique requires relatively good seeing conditions to be useful, and this is the reason the set of observed images was not of sufficient quality to have definite results.

The elegant simplicity of our SLM-based phase-diversity setup is evident from Figs. 3 and 4. The period of the diffraction grating is optimized to fit three diffraction orders in the CCD frame, the center one being the incoming light beam untouched, and the $+1(-1)$ diffraction order corresponding to $\mathrm{a}+x$ $(-x)$ phase-diverse image. Here, $x$ corresponds to the particular phase pattern embedded in the diffraction grating, which in our case is a vortex 1 phase or a defocus aberration. If this setup is used in traditional Zernike phase diversity, the SLM could be used to introduce astigmatism, spherical aberration, or any other aberration or combination of aberrations that best suit the particular telescope or atmospheric conditions. Consequently, it can be used as a substitute for the deformable mirror presently used in practically all adaptive optics systems. Whether using other aberrations apart from defocus improves the performance of the phase-diversity technique will be reported elsewhere. Another characteristic of our setup is that we have one nominal image, and two phase-diverse images to improve the confidence in the results (as explained in Paxman et al. 1992). However, since the phase-diversity algorithm can be implemented with an arbitrary number of phase-diverse images, it is possible to set up the SLM to produce more images and make the algorithm even more robust.

To illustrate the effect of the vortex pupil in an extended image as seen in Fig. 3, Fig. 5 shows the resulting image when a pinhole is placed at the F2 focal plane of THEMIS, as well as the modulation transfer function of a perfect vortex imaging system. The vortex donut shape of the point spread function (PSF) is evident, and the characteristic dip in the MTF is shown. The slight asymmetry in the PSF suggests some astigmatism in our post-focal instrument (see Jesacher et al. 2007). With good seeing conditions, the vortex phase-diverse images should show a blurring caused by the convolution of the object image with the donut-shaped PSF. Figures 3 and 5 represent the first time, to our

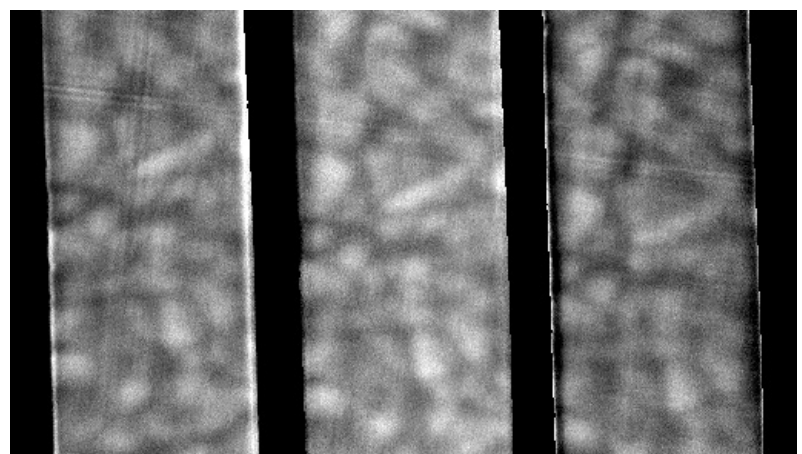

Fig. 4. Unfiltered full CCD images of defocus phase diversity. The central strip is the nominal image, and the left (right) strips the $-0.5 \lambda$ $(+0.5 \lambda)$ defocus phase-diverse images.
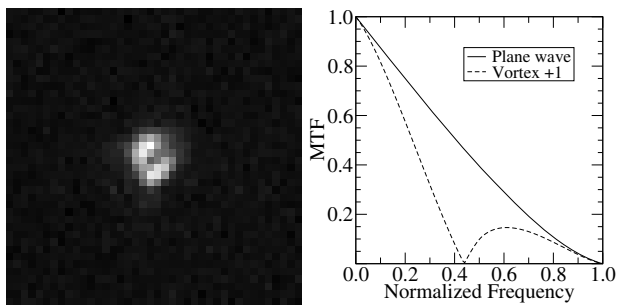

Fig. 5. (Left) Vortex +1 with the pinhole at F2. The donut shape is visible, but its light asymmetry suggests some astigmatism in the light path from F2 to the CCD plane. (Right) MTF of a perfect optical system (solid line) and perfect vortex optical system (dashed line).

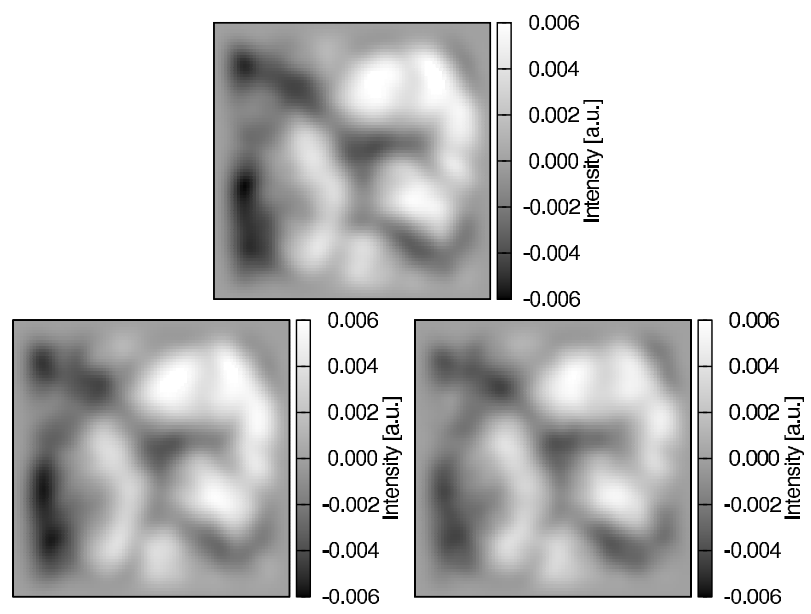

Fig. 6. $88 \times 88$ pixels field-of-view patch in the -1 (top), 0 (bottom left) and +1 (bottom right) vortex phase-diversities, after optimum filter.

knowledge, in which optical vortices (extended and point-like) are made with solar light.

As an example of the measurement process, we show one of the best $88 \times 88$ pixel, field-of-view patches of our observing campaign in Fig. 6, with its three phase diversities corresponding to $-1,0$ and +1 vortices. Each frame has been filtered by an optimum filter (see Bonet 1999). It is clear that the bad seeing significantly affected the image quality, to the point of limiting the frequencies present in the images to about half the diffraction limit. This is easily seen in Fig. 7, where the power spectra of the three frames are shown. Considering that we are digitally sampling at twice the optical diffraction limit frequency, the power spectra in good seeing should drop to zero near the edges of the images. If we refer to Fig. 5, we can see that the characteristic dip in the frequencies for a vortex pupil is lost in 


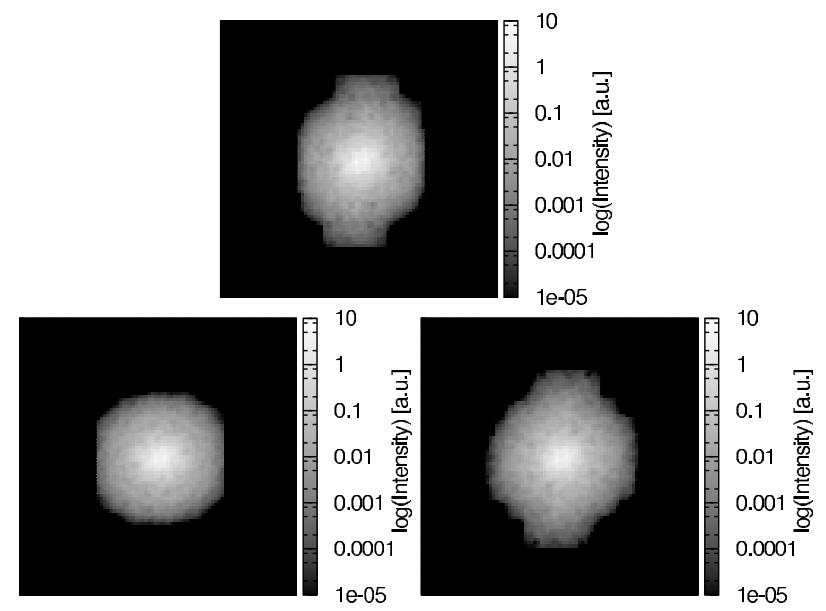

Fig. 7. Power spectra of the $88 \times 88$ pixels field-of-view patch of Fig. 6, for -1 (top), 0 (bottom left) and +1 (bottom right) vortex phasediversities, after optimum filter.

these conditions (for an example where the important frequencies survive, see Uribe-Patarroyo et al. 2010b), which limits the possibility of finding a vortex-like OTF in the phase-diverse images. Therefore, many combinations of aberrations can produce the power spectra shown, and the error metric has many local minima, making it impossible to determine the OTF with confidence. It is easy to see that good seeing is a necessary condition for phase-diversity measurements, so we are waiting for observing time with higher seeing quality, in order to obtain more solid results. One interesting conclusion from Fig. 7, however, is that the phase-diverse images preserved some higher frequencies than the nominal image. This implies that in bad seeing conditions the phase-diverse images could have a higher quality than the nominal image, if the phase diversity is appropriately chosen.

One of the strategies for taking advantage of this is to use a vortex phase and a defocus aberration as the phase diversity, as they are orthogonal aberrations (as explained in Uribe-Patarroyo et al. 2010a), one does not interfere with the other. In addition to this, more than three diffraction orders could be used, in which a matrix of phase-diverse images is obtained with horizontal vortex orders and vertical defocus orders. Then, having a larger array of phase diversities could raise the possibility of having some phase-diverse images with good quality and high-frequency content, in exchange for dividing the available photon budget between many diffraction orders.

\section{Conclusions}

We designed and assembled a system for measuring the OAM spectrum of solar photons. The system, based on a phasediversity technique, works with extended images and provides information about the mean OAM spectrum of a solar region.
However, definite results were not obtained during the 2009 observing campaign due to poor seeing conditions. Apart from awaiting observing time with higher quality, some strategies for bad seeing conditions have been proposed.

Acknowledgements. A.A.R. and R.M.S. acknowledge financial support from the Spanish MICINN through the project AYA2007-63881

\section{References}

Agarwal, G. S. 1999, J. Opt. Soc. Am. A, 16, 2914

Akamatsu, D., \& Kozuma, M. 2003, Phys. Rev. A, 67, 023803

Allen, L., Beijersbergen, M. W., Spreeuw, R. J. C., \& Woerdman, J. P. 1992, Phys. Rev. A, 45, 8185

Berkhout, G. C. G., \& Beijersbergen, M. W. 2009, J. Opt. A: Pure and Appl. Opt., 11, 094021

Bogatyryova, G. V., Fel'de, C. V., Polyanskii, P. V., et al. 2003, Opt. Lett., 28, 878

Bonet, J. A. 1999, in Motions in the Solar Atmosphere, ed. A. Hanslmeier, \& M. Messerotti, A\&SS Library, 239, 1

Danchi, W. C., Allen, R. J., Benford, D. J., et al. 2004, in SPIE Conf. Ser. 5491, ed. W. A. Traub, 236

del Toro Iniesta, J. C. 2003, Introduction to Spectropolarimetry (Cambridge, UK: Cambridge University Press)

Elias, II, N. M. 2008, A\&A, 492, 883

Franke-Arnold, S., Allen, L., \& Padgett, M. 2008, Laser \& Photonics Review, 2, 299

Gbur, G., \& Tyson, R. K. 2008, J. Opt. Soc. Am. A, 25, 225

Gibson, G., Courtial, J., Padgett, M., et al. 2004, Opt. Express, 12, 5448

Gonsalves, R. A., \& Chidlaw, R. 1979, in SPIE Conf. Ser. 207, ed. A. G. Tescher, 32

Harwit, M. 2003, ApJ, 597, 1266

Jackson, J. D. 1962, Classical Electrodynamics (New York: Wiley)

Jenkins, C. 2008, MNRAS, 384, 515

Jesacher, A., Schwaighofer, A., Fürhapter, S., et al. 2007, Opt. Express, 15, 5801 Leach, J., Padgett, M. J., Barnett, S. M., Franke-Arnold, S., \& Courtial, J. 2002, Phys. Rev. Lett., 88, 257901

Liu, Y., Gao, C., Qi, X., \& Weber, H. 2008, Opt. Express, 16, 7091

Löfdahl, M. G., \& Scharmer, G. B. 1994, A\&AS, 107, 243

Mair, A., Vaziri, A., Weihs, G., \& Zeilinger, A. 2001, Nature, 412, 313

Palacios, D. M., Maleev, I. D., Marathay, A. S., \& Swartzlander, Jr., G. A. 2004, Phys. Rev. Lett., 92, 143905

Paterson, C. 2005, Phys. Rev. Lett., 94, 153901

Paxman, R. G., Schulz, T. J., \& Fienup, J. R. 1992, J. Opt. Soc. Am. A, 9, 1072 Ponomarenko, S. A. 2001, J. Opt. Soc. Am. A, 18, 150

Rayrole, J., \& Mein, P. 1993, in The Magnetic and Velocity Fields of Solar Active Regions, ed. H. Zirin, G. Ai, \& H. Wang, IAU Colloq., 141, ASP Conf. Ser., 46,170

Serabyn, G., Mawet, D., \& Burruss, R. 2010, in AAS Meeting Abstracts, 215, 377.06

Solanki, S. K. 1993, Space Sci. Rev., 63, 1

Solanki, S. K. 2003, A\&ARev, 11, 153

Stenflo, J. O. 1994, Solar Magnetic Fields. Polarized Radiation Diagnostics (Dordrecht: Kluwer Academic Publishers)

Swartzlander, Jr., G. A. 2001, Opt. Lett., 26, 497

Tyler, G. A., \& Boyd, R. W. 2009, Opt. Lett., 34, 142

Uribe-Patarroyo, N., Alvarez-Herrero, A., \& Belenguer, T. 2010a, Opt. Express, 18,21111

Uribe-Patarroyo, N., Alvarez-Herrero, A., \& Belenguer, T. 2010b, Phys. Rev. A, 81,053822

Vasnetsov, M. V., Pas'ko, V. A., \& Soskin, M. S. 2005, New J. Phys., 7, 46

Vicari, L., ed. 2003, Optical Applications of Liquid Crystals, Series in Optics and Optoelectronics (London: Institute of Physics Publishing)

Yi-Xin, Z., \& Ji, C. 2009, Chinese Phys. Lett., 26, 074220

Zambrini, R., \& Barnett, S. M. 2006, Phys. Rev. Lett., 96, 113901 